\title{
Stereoselective Discrimination and Quantification of Arginine and N-Blocked Arginine Enantiomers by Formation and Dissociation of Calcium-Mediated Diastereomeric Trimer Complexes with a Chiral Reference Compound Using Electrospray Ionization-Ion Trap Tandem Mass Spectrometry
}

\author{
Kevin A. Schug and Wolfgang Lindner \\ Department of Analytical Chemistry, University of Vienna, Vienna, Austria
}

Chiral resolution of arginine (Arg) and Arg derivatives is demonstrated using electrospray ionization-tandem mass spectrometry (ESI-MS). Calcium ion $\left(\mathrm{Ca}^{\mathrm{II}}\right)$-mediated trimeric clusters are generated, which incorporate the analyte of interest and an enantiomerically pure reference molecule of similar metal ion affinity. Two methods, one based on the measurement of a competitive-dissociation-based branching ratio $\left(\mathrm{R}_{\text {chir }}\right)$ by the kinetic method $(\mathrm{KM})$ and one based on the measurement of a chiral recognition ratio (CR) by a similar method, are compared. Incorporating N-blocked Arg derivatives (Z-Arg and Boc-Arg) as chiral references provides chiral resolution greater than that previously reported for Arg enantiomers. In a reciprocal manner, pure Arg enantiomers can be used as references for discriminating enantiomers of these N-blocked Arg derivatives. Condensed-phase and gas-phase $\mathrm{Ca}^{\mathrm{II}}$ ion affinity relative to Arg is also addressed qualitatively for other acidic, basic, and neutral amino acids. In some cases, when only one offspring ion is observed (insufficient for KM analysis), the $\mathrm{CR}$ method can be applied as an alternative to obtain a measurable stereoselectivity value for the system. The results of these experiments demonstrate the applicability of, and the difference between, the KM and the $\mathrm{CR}$ method for improved quantitative analysis of enantiomeric excess for Arg. (J Am Soc Mass Spectrom 2005, 16, 825-834) (C) 2005 American Society for Mass Spectrometry

$\mathrm{T}$ The phenomenon of chirality, or molecular asymmetry, was first reported by Louis Pasteur in 1848 with the crystallographic analysis of tartaric acid [1]. Since this seminal work, the importance of chiral molecules, particularly of enantiomerically pure chiral molecules, has been recognized and manifested in countless pharmaceutical and biological advancements. With the necessity for the development of enantiomerically pure drugs has arisen a need for complementary analytical methodologies. Such analyses rely on the incorporation of a chiral environment to differentiate enantiomers through the formation of distinguishable diastereomers via multi-point interactions. A variety of effective methods have been developed, including many spectro-

Published online March 30, 2005

Address reprint requests to Dr. W. Lindner, Department of Analytical Chemistry, University of Vienna, Währingerstrasse 38, A-1090 Vienna, Austria. E-mail: wolfgang.lindner@univie.ac.at scopic and chromatographic techniques [2]. With the advent of high-throughput drug development, faster, more efficient, and robust alternatives for evaluating enantiomeric purity are continually being sought and applied. MS- and tandem MS-based methods, particularly in combination with electrospray ionization (ESI), offer the ability to fill this niche; also adding the advantage of removal of matrix and possible solvent effects prior to quantification [3].

Several comprehensive reviews have been published on the use of MS for the analysis of enantiomers [4-6]. A common thread of relevant works, because of the nonchiral environment present in a mass spectrometer, is the necessity to incorporate chirality through chiral reference molecules. For discrimination, analyte enantiomers should interact with the chiral reference molecule to form differentially bound $(\Delta \Delta \mathrm{G} \neq 0)$ diastereomeric complexes. Currently, there are five well-defined approaches established in the literature [7, 8]. Briefly, 


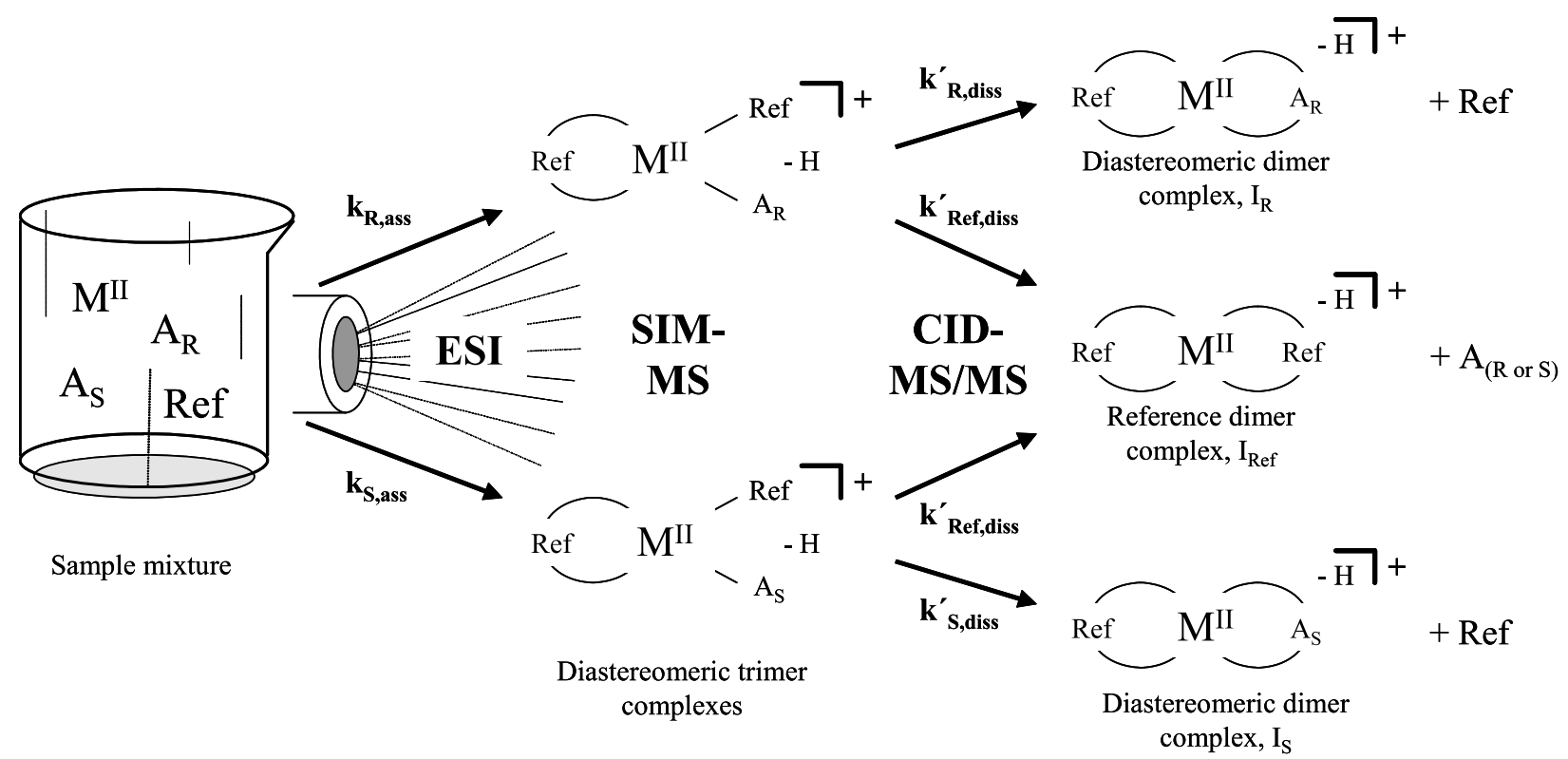

Figure 1. Schematic depicting the formation of metal-mediated diastereomeric trimer complex ions followed by their competitive unimolecular dissociation to dimeric complex ions using ESI-tandem MS. $M^{I I}$, Ref, $A_{R}$ and $A_{S}$ denote metal mediator, enantiomerically pure reference, and analyte enantiomers, respectively.

these can be classified as: (1) Host-guest methods incorporating, but not limited to, competitive binding and isotopic labeling experiments [9, 10]; (2) ion-molecule exchange reactions to measure rates of guest exchange in gas-phase experiments [11-14]; (3) kinetic resolution in solution followed by MS analysis [15]; (4) collisioninduced dissociation (CID) of diastereomeric adducts [16-20]; and (5) the kinetic method (KM) [7, 8, 21, 22].

The KM was first described by Cooks and Kruger in 1977 for use in determining proton affinities of alkylamines [23]. Since then, it has been applied to both thermodynamic (e.g., proton or metal ion affinity and gas-phase basicity) [24-27] measurements and steric (chiral and isomeric discrimination) $[7,8,21,28,29]$ determinations. For chiral discrimination, the parent cluster ion of interest contains an analyte enantiomer $\left(A_{x}\right)$, an enantiomerically pure reference molecule (Ref), and a metal ion $(\mathrm{M})$ in a trimeric arrangement around the metal center $\left(\left[\mathrm{A}_{\mathrm{X}} \operatorname{Ref}_{2} \mathrm{M}-\mathrm{H}\right]^{+}\right)$. Discrimination between two different enantiomers is achieved by measurement of the unimolecular dissociation pathways of the diastereomeric trimer ion to form the chiral dimer ions, $\left[\mathrm{A}_{\mathrm{X}} \operatorname{RefM}-\mathrm{H}\right]^{+}$and $\left[\operatorname{Ref}_{2} \mathrm{M}-\mathrm{H}\right]^{+}$(see Figure 1). From the dimer ion signals, $\left(\mathrm{I}_{\mathrm{R}}, \mathrm{I}_{\mathrm{S}}\right.$, and $\left.\mathrm{I}_{\mathrm{Ref}}\right)$ branching ratios can be calculated.

The choice of metal ion and reference is of prime importance for establishing a system which conforms to $\mathrm{KM}$ analysis. If the reference and analyte do not have similar metal ion affinity, the requisite dissociation channels are either not observed or ion abundances are too low to provide reliable measurements. In such a case, an alternative method based on the measurement of a "chiral recognition ratio" (CR) can be substituted [19].
The measurement of CR is based, similar to the KM, on the dissociation of a diastereomeric complex. Instead of the ratio of product ions resulting from dissociation, calculation of CR relies on the ratio of the intensity of one fragment ion to that of the parent ion. In general, accurate enantiomeric excess analyses (enantiomeric purity measurements) have been reported using this technique $[19,20]$. However, proponents of the KM have argued the inferiority of the CR method [22].

In this work, chiral discrimination of arginine (Arg) and N-protected arginine derivatives using mainly $\mathrm{Ca}^{\mathrm{II}}$ mediation is demonstrated. Arg is known to be a precursor to nitric oxide production and has been administered as an oral food supplement to reduce hypertension, increase lipid metabolism, and increase growth hormone-releasing agents [30, 31]. The N-protected Arg derivatives provide a set of model compounds for mimicking the behavior of a C-terminal Arg unit, a common product of tryptic digests. Thus, the development of a method to assess enantiomeric purity of these molecule types, as well as studying the underlying mechanism behind their interaction and affinity to metals in the context of diastereomeric cluster formation, is a worthwhile undertaking.

The difficulty of analyzing Arg by the KM, because of its exceptionally high gas-phase metal ion affinity, has been previously reported [21]. To partially address this complication, a method has recently been reported for the isomeric discrimination of arginine-containing dipeptide molecules by the KM using similar argininecontaining dipeptides as references [29]. To compliment this approach in the current work, chiral discrimination of Arg and Arg-derived analytes using the complemen- 
tary Arg and Arg-derivatives as enantiomerically pure reference molecules with similar metal ion affinity is shown. Comparison of the results with other enantiomerically pure amino acid molecules of varying functionality is shown to reinforce this approach. The calculation of the CR is evaluated where the KM cannot be applied. Also, an overall comparison of the two analytical methods is offered for many of the analyte/reference systems tested. Finally, quantitative discrimination of Arg enantiomers is demonstrated through the construction of a calibration curve with sample mixtures of defined enantiomeric excess, for both methods.

\section{Experimental}

\section{Instrumental}

Experiments were performed on an Agilent 1100 Series LC/MSD SL ion trap mass spectrometer (Agilent Technologies, Vienna, Austria) with an electrospray ionization interface. Sample solutions were introduced via a syringe pump operating at $5 \mu \mathrm{L} / \mathrm{min}$. The electrospray needle voltage, operating in the positive ionization mode, was optimized at $4205 \mathrm{~V}$ for the formation of metal mediated trimer ions. Nebulizer gas was supplied at 5 psi and drying gas was operated at $3.0 \mathrm{~L} / \mathrm{min}$ at a temperature of $250{ }^{\circ} \mathrm{C}$. The exit of the desolvation capillary was set to $165 \mathrm{~V}$; and the skimmer cone to $40 \mathrm{~V}$. The ion lenses and octapole were also optimized to provide the maximum trimer ion signal. The ion trap was operated in partial full scan MS/MS (200-1100 m/z) mode to monitor the breakdown of the trimer ion. Isolation of the parent trimer ion is achieved by applying multiple waveforms in the trap to eject all undesired ions. An AC potential is then placed on the parent ion with variable energy to affect fragmentation of the parent into the dimeric offspring ions [28]. The excitation time used was $40 \mathrm{~ms}$ and the excitation amplitude was varied from between 0.60 and $1.00 \mathrm{~V}$. The "Smartfrag" setting in the ion trap software was turned off to maintain a constant excitation amplitude. An excitation amplitude was chosen for each different reference/analyte system to achieve a moderate intensity and a steady signal for offspring ions. Analyses were performed in triplicate (n $=3$ ) for each mixture and data reported for each run is the average of 60 spectral scans, where each scan is the average of five microscans.

\section{Chemicals and Sample Mixtures}

All sample mixtures were prepared for analysis in a 50:50 methanol/water mixture [HPLC grade methanol from Fisher Chemicals (Schwerte, Germany) and Ultrapure LCMS grade water from Fluka (Buchs, Switzerland)]. Final sample mixtures were composed of $50 \mu \mathrm{mol} / \mathrm{L}$ $(\mu \mathrm{M})$ each of the analyte and reference molecules $(1: 1$ stoichiometry) and $12.5 \mu \mathrm{M}$ of metal ion. Pure $(R)$ and (S) enantiomers of arginine (Arg), N $\alpha$-blocked benzyloxycarbonyl-arginine (Z-Arg), N $\alpha$-blocked tert-bu- tyloxycarbonyl-arginine (Boc-Arg), lysine (Lys), histidine (His), glutamic acid (Glu), and phenylalanine (Phe) were purchased from Bachem (Weil am Rhein, Germany) and used without further purification. $\mathrm{N} \alpha$ blocked acetyl-(S)-arginine (Ac-Arg) and C-blocked (S)arginine amide $\left(\mathrm{Arg}-\mathrm{NH}_{2}\right)$ were also purchased from Bachem. N-acetyl-(S)-arginine-amide (Ac-Arg- $\left.\mathrm{NH}_{2}\right)$ was synthesized from (S)-Arg- $\mathrm{NH}_{2}$ and purified with ion exchange chromatography in house. Metal ion salts screened in this study for evaluation of metal mediator were $\mathrm{Cu}(\mathrm{II})$ acetate, $\mathrm{Zn}$ (II) acetate, $\mathrm{Ni}$ (II) acetate, and $\mathrm{CaCl}_{2}$, all obtained from Merck (Darmstadt, Germany), and $\mathrm{MgCl}_{2}$, was obtained from Fluka.

Preliminary experiments were performed to screen for the best metal ion for discrimination of Arg as analyte, using the $\mathrm{N}$ - and C-blocked Arg derivatives as references, and vice versa. Chiral discrimination by the $\mathrm{KM}$ was only observed with $\mathrm{Ca}^{\mathrm{II}}$ - and $\mathrm{Mg}^{\mathrm{II}}$-mediated trimer ions. Therefore, results obtained for the other metal ion systems are largely omitted. Results below are shown for the systems analyzed in experiments for Arg and Arg derivatives using $\mathrm{Ca}^{\mathrm{II}}$ - and $\mathrm{Mg}^{\mathrm{II}}$-mediation (interchanging references and analytes). The use of other amino acid molecules (with different functional moieties) as references for Arg discrimination with $\mathrm{Ca}^{\mathrm{II}}$, were included only in the studies with $\mathrm{Ca}^{\mathrm{II}}$ mediation for the purpose of comparing gasand condensed-phase metal ion affinity. Similarly, calculation of CR values was performed only where relevant KM data was collected.

\section{Results and Discussion}

\section{Chiral Discrimination}

The goal of this work was to develop a tandem MS approach for the discrimination and quantification of Arg amino acid enantiomers. To accomplish this, both the $\mathrm{KM}$ and the $\mathrm{CR}$ method were investigated. The high metal affinity of the guanidinium unit in Arg has previously hindered the finding of a quantitative KMbased metal/reference system to do so [21, 32]. A series of commonly employed metal ion mediators were screened along with a few Arg-derived references molecules. The N- and C-blocked Arg derivatives investigated (Ac-Arg, Arg- $\mathrm{NH}_{2}$, Ac-Arg- $\mathrm{NH}_{2}, \mathrm{Z}-\mathrm{Arg}$, and BocArg) were chosen because they were readily available in enantiomerically pure (R) or (S) forms and because they were believed to provide two characteristics essential to being a good KM reference molecule: (1) they should have a similar metal ion affinity to Arg, and (2) the placement of additional groups on their molecular scaffold should add steric information favoring chiral discrimination. Since the ionic sites likely constitute the metal ion binding sites of the molecules, information related to dominating metal-coordinating groups in the molecule could be evaluated through systematic variation of chemically modified ( $\mathrm{N}$ - and C-terminal blocking) ionic binding sites.

The formation and dissociation of metal-mediated 
Table 1. Measured chiral discrimination values $\left(\mathrm{R}_{\text {chir }}(\mathrm{KM})\right.$ and $\left.\mathrm{CR}\right)$ for $\mathrm{Ca}^{\mathrm{II}}$ - and $\mathrm{Mg}^{\mathrm{II}}$-mediated analyte and reference systems; focusing on the determination for Arg and N-blocked Arg derivatives

\begin{tabular}{|c|c|c|c|c|c|c|}
\hline $\begin{array}{c}\text { Analyte } \\
((R) \text { vs. }(S))\end{array}$ & Reference & $\mathrm{R}_{\text {chir }}\left(\mathrm{Ca}^{\prime \prime}\right)$ & $\mathrm{R}_{\text {chir }}\left(\mathrm{Mg}^{\prime \prime}\right)$ & CR (Ca") & CR (Mg") & Method? ${ }^{c}$ \\
\hline \multirow[t]{12}{*}{ Arg } & Z-(R)-Arg & $2.2 \pm 0.1$ & $1.19 \pm 0.06$ & $0.37 \pm 0.02$ & $0.88 \pm 0.02$ & no pref \\
\hline & Z-(S)-Arg & $0.47 \pm 0.02$ & $1.06 \pm 0.06$ & $0.42 \pm 0.02$ & $0.89 \pm 0.05$ & no pref \\
\hline & Boc- $(R)-\operatorname{Arg}$ & $1.04 \pm 0.05$ & $0.90 \pm 0.02$ & $0.97 \pm 0.04$ & $1.22 \pm 0.04$ & no pref \\
\hline & $\operatorname{Ac}-(S)-\operatorname{Arg}$ & $1.02 \pm 0.02$ & & $1.07 \pm 0.02$ & & no pref \\
\hline & (S)-Arg- $\mathrm{NH}_{2}$ & $\mathrm{NT}^{\mathrm{a}}$ & & & & \\
\hline & $\mathrm{Ac}-(S)-\mathrm{Arg}-\mathrm{NH}_{2}$ & NT & & & & \\
\hline & $(R)$-His & $0.73 \pm 0.05$ & & $1.10 \pm 0.02$ & & KM \\
\hline & (S)-His & $0.70 \pm 0.03$ & & $1.03 \pm 0.02$ & & $\mathrm{KM}$ \\
\hline & $(R)$-Glu & $6 \pm 3$ & & $0.67 \pm 0.02$ & & $\mathrm{CR}$ \\
\hline & (S)-Glu & $2.3 \pm 0.9$ & & $0.77 \pm 0.03$ & & $\mathrm{CR}$ \\
\hline & $(R)$-Lys & $N D^{b}$ & & $1.11 \pm 0.07$ & & $\mathrm{CR}$ \\
\hline & $(R)$-Phe & ND & & & & \\
\hline \multirow{5}{*}{ Z-Arg } & $(R)-\operatorname{Arg}$ & $0.44 \pm 0.04$ & $0.96 \pm 0.05$ & $1.03 \pm 0.04$ & $0.84 \pm 0.01$ & KM \\
\hline & (S)-Arg & $2.2 \pm 0.3$ & & $1.07 \pm 0.02$ & & KM \\
\hline & Boc- $(R)-\operatorname{Arg}$ & $0.79 \pm 0.04$ & NT & $0.89 \pm 0.03$ & & KM \\
\hline & Boc-(S)-Arg & $1.28 \pm 0.06$ & & $0.88 \pm 0.03$ & & KM \\
\hline & Ac- $(S)-\operatorname{Arg}$ & $0.97 \pm 0.07$ & & $0.75 \pm 0.05$ & & $\mathrm{CR}$ \\
\hline \multirow[t]{4}{*}{ Boc-Arg } & $(R)-\operatorname{Arg}$ & $0.70 \pm 0.07$ & $0.68 \pm 0.06$ & $1.24 \pm 0.03$ & $0.80 \pm 0.03$ & $\mathrm{KM}$ \\
\hline & (S)-Arg & $1.6 \pm 0.2$ & $1.5 \pm 0.2$ & $0.87 \pm 0.01$ & $0.98 \pm 0.04$ & KM \\
\hline & Z-(R)-Arg & $0.98 \pm 0.04$ & NT & $0.84 \pm 0.03$ & & $\mathrm{CR}$ \\
\hline & Ac-(S)-Arg & $0.690 \pm 0.007$ & & $1.33 \pm 0.04$ & & $\mathrm{KM}$ \\
\hline His & $(R)-\operatorname{Arg}$ & $1.2 \pm 0.2$ & & $0.9 \pm 0.2$ & & no pref \\
\hline Glu & $(R)-\operatorname{Arg}$ & $1.0 \pm 0.6$ & & $0.7 \pm 0.4$ & & no pref \\
\hline Phe & (R)-Arg & $1.5 \pm 0.9$ & & $0.6 \pm 0.4$ & & no pref \\
\hline Lys & $(R)$-Arg & ND & & & & \\
\hline
\end{tabular}

${ }^{a}$ NT denotes that no trimer ion was able to be isolated for fragmentation.

${ }^{b}$ ND denotes that the signal of only one dimer ion was observed and no KM branching ratio could be measured.

"Method of choice: no pref indicates no difference between methods; KM denotes better discrimination by the KM; and CR denotes better discrimination by the CR technique. A blank cell indicates no measurement was recorded.

diastereomeric clusters in the context of applying the calculations below based on the $\mathrm{KM}$ and the CR are shown in Figure 1. In the KM, the competitive dissociation of the trimer ions to form dimer ions provides a route for measuring the free energy difference between the diastereomeric systems based on each analyte enantiomer. The ratio of intensities of the dimeric complex ions, called the branching ratio $R_{X}$ (where $X$ represents a general designation for $(R)$ - or $(S)$-enantiomers), resulting from unimolecular dissociation by CID of the trimer complex ion, is expressed by eq 1 [33]:

$$
R_{X}=\frac{\left[M(\operatorname{Ref})\left(A_{X}\right)-H\right]^{+}}{\left[M(\operatorname{Ref})_{2}-H\right]^{+}} .
$$

The relative branching ratio, $R_{\text {chir }}$ is then used to describe the degree of chiral discrimination by:

$$
\begin{aligned}
R_{\text {chir }} & =\frac{R_{R}}{R_{S}} \\
& =\frac{\left[M(\operatorname{Ref})\left(A_{R}\right)-H\right]^{+}}{\left[M(\operatorname{Ref})_{2}-H\right]^{+}} / \frac{\left[M(\operatorname{Ref})\left(A_{S}\right)-H\right]^{+}}{\left[M(\operatorname{Ref})_{2}-H\right]^{+}} .
\end{aligned}
$$

A nonunity $\mathrm{R}_{\text {chir }}$ value indicates chiral discrimination in the system based on the presence, isolation, and fragmentation of the specific trimer ion of interest and the subsequent presence of the requisite dimer ion forms for calculation.

Measurement of the CR has been presented in detail previously by Yao and coworkers $[19,20]$. Similar to the $\mathrm{KM}$, this technique relies on the formation, isolation, and fragmentation of a diastereomeric cluster ion composed of a chiral analyte of interest and an enantiomerically pure reference. The dissociation of the specified diastereomeric cluster ion leads to the loss of one reference stereoisomer from the cluster. The CR values measured in this study (in coherence with the nomenclature described previously for the KM and Figure 1) are thus given by:

$$
\begin{aligned}
C R= & \left(\frac{\left[M\left(\operatorname{Ref}_{\mathrm{R}}\right)\left(\mathrm{A}_{\mathrm{S}}\right)-\mathrm{H}\right]^{+}}{\left[\mathrm{M}\left(\operatorname{Ref}_{\mathrm{R}}\right)_{2}\left(\mathrm{~A}_{\mathrm{S}}\right)-\mathrm{H}\right]^{+}}\right) / \\
& \left(\frac{\left[\mathrm{M}\left(\operatorname{Ref}_{\mathrm{R}}\right)\left(\mathrm{A}_{\mathrm{R}}\right)-\mathrm{H}\right]^{+}}{\left[\mathrm{M}\left(\operatorname{Ref}_{\mathrm{R}}\right)_{2}\left(\mathrm{~A}_{\mathrm{R}}\right)-\mathrm{H}\right]^{+}}\right)
\end{aligned}
$$

For the purpose of direct comparison of CR values with $R_{\text {chir }}$ values, identical data sets were evaluated with the two methods.

The results of the experiments using $\mathrm{Ca}^{\mathrm{II}}$ metal mediation and a variety of references for both the KM 
(a) $(R)-A r g+Z-(R)-A r g+C a^{\prime \prime}$

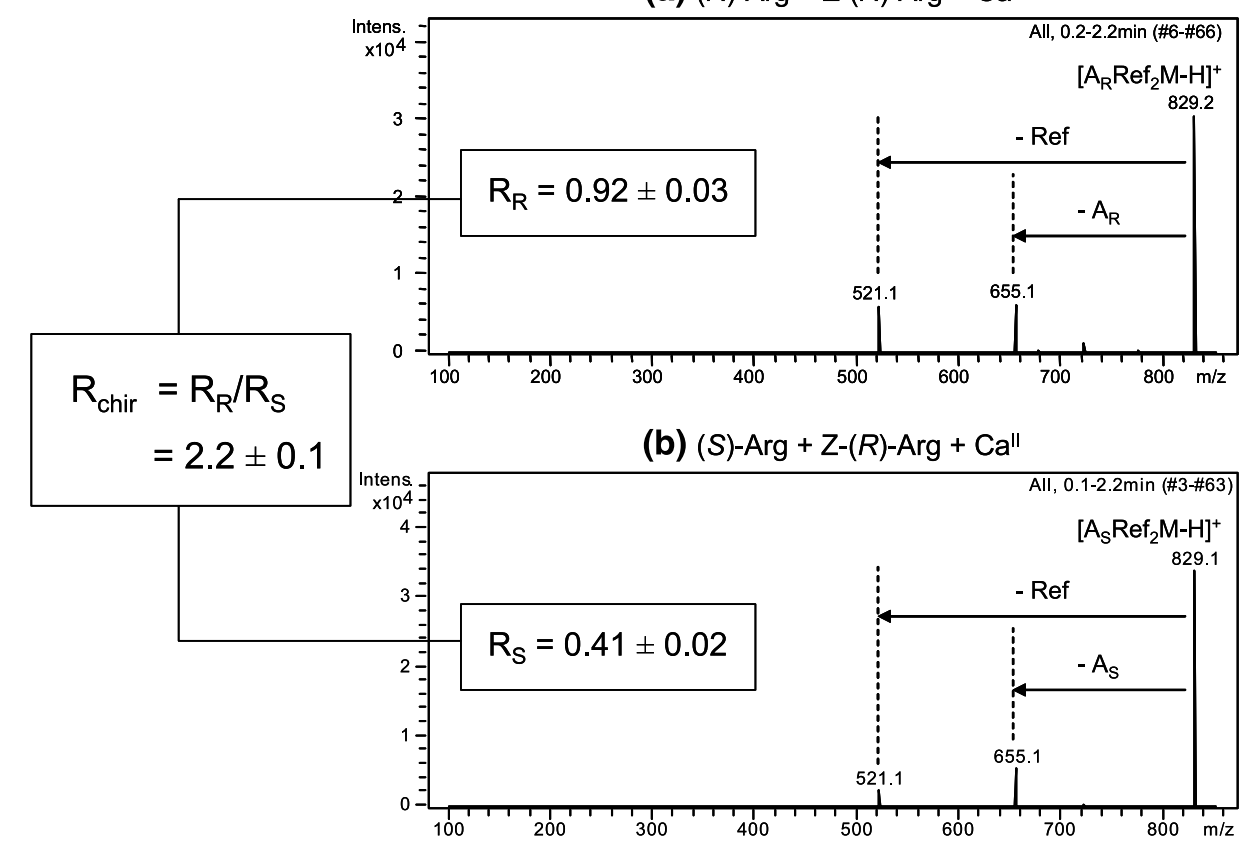

Figure 2. Example of chiral discrimination of Arg by Z-(R)-Arg using the KM. (a) and (b) show the dissociation of the trimer ion $(\mathrm{m} / \mathrm{z}=829)$ formed from $(R)$-Arg and $(S)$-Arg, respectively, with $\mathrm{Z}-(\mathrm{R})-\mathrm{Arg}$ and $\mathrm{Ca}^{\mathrm{II}}$ metal mediation.

and CR approaches are shown in Table 1. Values for $\mathrm{Mg}^{\mathrm{II}}$ mediation are presented as a comparison for the more promising systems investigated based on the measured KM values. Also, the best method (KM versus $\mathrm{CR}$ ) for each analyte/reference system is indicated. Results from the KM approach are presented here first.

The greatest degree of $R_{\text {chir }}$ for Arg enantiomer discrimination reported previously utilized $\mathrm{Ni}^{\mathrm{II}}$ mediation and (S)-Lys as a reference $\left(\mathrm{R}_{\text {chir }}=0.68\right)$ [34]. Here, using Z-Arg as a reference and $\mathrm{Ca}^{\mathrm{II}}$ mediation, values less than 0.5 and greater than 2.0 are measured $\left(R_{\text {chir }}=\right.$ $0.47 \pm 0.02$ for Z-(S)-Arg and $2.2 \pm 0.1$ for Z-(R)-Arg, respectively) for discrimination of Arg enantiomers. Figure 2 shows an example of the KM measurement and calculation of $R_{\text {chir }}$ for Arg based on using Z-(R)Arg as a reference. The other blocked Arg references were unsuccessful at discriminating between the two enantiomers. The lack of favorable discrimination using Boc-Arg is particularly surprising, considering the increased steric bulk of the N-terminal carbamate-bound tert-butyloxycarbonyl group compared to that of the benzyloxycarbonyl group in Z-Arg [35]. It has been previously reported, however, that the presence of a $\pi$-rich benzyl ring in the reference molecule can increase the measured resolution in KM-based experiments $[29,34]$.

In a reciprocal study, Arg enantiomers and the remaining Arg-derivative enantiomers were examined as references for discriminating Z-Arg and Boc-Arg enantiomer analytes. For Z-Arg analytes, both Arg and Boc-Arg were efficient chiral references when using $\mathrm{Ca}^{\mathrm{II}}$ mediation. The values acquired using Arg as a reference $\left[R_{\text {chir }}=0.44 \pm 0.04\right.$ for $(R)$-Arg and $2.2 \pm 0.3$ for (S)-Arg] were very similar to those acquired above when the roles of the molecules in the system were reversed. Boc-Arg was a less efficient reference, although chiral discrimination of Z-(R)- and Z-(S)-Arg was still measured. Ac-Arg was not a suitable reference. When the same methodology was applied to distinguish between Boc-Arg enantiomers, Arg and, surprisingly, Ac-Arg provided sufficient resolution as references with $\mathrm{Ca}^{\mathrm{II}}$ mediation, whereas Z-Arg was a poor reference.

For the systems incorporating Arg and Arg-derivatives, excluding C-blocked derivatives, comparable $\mathrm{KM}$ determinations were made with $\mathrm{Mg}^{\mathrm{II}}$ mediation (see Table 1). $\mathrm{Mg}^{\mathrm{II}}$ is smaller than $\mathrm{Ca}^{\mathrm{II}}$, and as such, might be expected to bind more strongly to the ligands because of its higher charge density. In reality, the amplitude voltages necessary to fragment the $\mathrm{Mg}^{\mathrm{II}}$-centered trimer ions were significantly less than those used with $\mathrm{Ca}^{\mathrm{II}}$. This is likely due to the small metal ion being less stably solvated (complexed) by the three ligands (four functional units, see next section), compared with the larger $\mathrm{Ca}^{\mathrm{II}}$ ion. With the exception of the discrimination of Boc-Arg using Arg as a reference and vice versa, the $R_{\text {chir }}$ values recorded using the $\mathrm{KM}$ with $\mathrm{Mg}^{\mathrm{II}}$ mediation were not as pronounced as those recorded with $\mathrm{Ca}^{\mathrm{II}}$. To the best of our knowledge, enantiomer discrimination of Boc-Arg by Arg using $\mathrm{Mg}^{\mathrm{II}}$ mediation $\left(\mathrm{R}_{\text {chir }}=0.68 \pm\right.$ 0.06 for $(R)$-Arg and $R_{\text {chir }}=1.5 \pm 0.2$ for $(S)$ - Arg) is the first report of using this metal ion for chiral discrimination by the KM. For reciprocal systems incorporating 
only Boc-Arg and Z-Arg with Mg ${ }^{\mathrm{II}}$ mediation, no isolable trimer ion signal could be measured. Collectively, these results indicate that there likely exists a favorable ligand-metal ion size relationship for optimizing/tuning discrimination in these and similar systems.

Comparisons between discrimination of enantiomers based on the KM and the CR MS/MS-based approaches show some interesting similarities as well as differences. In general, for these analytes, references, and metals, it is difficult to say whether one method performed better than the other. This is indicated by the last column in Table 1. The obvious situation where the $\mathrm{CR}$ method has a decided advantage over the KM is when only one product ion, or only a very low intensity second product ion, is observed upon fragmentation of the metal-mediated diastereomeric trimer ions. In these cases, where Glu, Lys, and Phe were used as chiral references for discrimination of Arg enantiomers, the dimeric forms incorporating one Arg analyte and one reference molecule were the dominant offspring ions. The subsequent $\left[\mathrm{M}(\operatorname{Ref})_{2}-\mathrm{H}\right]^{+}$molecule was either not detected (for Lys and Phe) or detected in extremely low abundance (for Glu). Therefore, the subsequent KM measurement either could not be made or had an extremely high error associated with it. In contrast, CR values could be measured in these cases and in some cases, particularly for Glu references, acceptable values of enantiomeric discrimination were recorded. In general, differences in the successes of the two methods appear to stem from reference/metal relative affinity issues. The KM is expected, in the majority of cases, to have superior reproducibility because of the presence of a common homochiral dimeric reference ion to normalize each measurement. Still, more than acceptable $\%$ RSD values of $1.4 \%$ have been previously reported using the CR technique [19]. In these experiments, the $\%$ RSD of the measured values for each method are similar.

\section{$C a^{I I}$-Mediation}

The fact that $\mathrm{Ca}^{\mathrm{II}}$ performed better than all of the other metal ions screened is interesting. Compared with the transition metal ions investigated, $\mathrm{Ca}^{\mathrm{II}}$ does not possess the accessible $d$-orbital valency that is commonly associated with specific geometric arrangements of ligands around metals such as $\mathrm{Cu}^{\mathrm{II}}, \mathrm{Ni}^{\mathrm{II}}$, and $\mathrm{Zn}^{\mathrm{II}}$. Still, reproducible KM measurements for chiral discrimination, demonstrated by this work, can be performed. Previous studies in the literature have shown various affinities by different amino acids and amino acid residues for the calcium ion. These studies report strong affinity for acidic amino acids and residues, such as aspartic acid (Asp), glutamic acid (Glu), and C-terminal units [36, 37], as well as a slight preference for His-rich peptides [38]. Gross and Nemirovsky used the KM specifically to investigate the $\mathrm{Ca}^{\mathrm{II}}$ affinity of analogs of rabbit skeletal troponin $\mathrm{C}$ [27]. They found a significant difference in $\mathrm{Ca}^{\mathrm{II}}$ binding related to the number of Asp residues present and the conformation of the peptides studied.

To qualitatively study the relative condensed- and gas-phase affinities for $\mathrm{Ca}^{\mathrm{II}}$ of other amino acids compared to Arg, a series of amino acids (Glu, His, and Phe as representative acidic, basic, and neutral amino acids, respectively) were chosen to be included in this study. The pure enantiomers of these molecules were tested as both analytes and references in a system containing $\mathrm{Ca}^{\mathrm{II}}$ and Arg. The results are given in Table 1. His and Glu were able to act as sufficient references to distinguish between Arg enantiomers using the KM, even though the values measured for Glu suffered from extremely bad precision. With respect to chiral discrimination in the other systems, the results were poor, generally exhibiting $R_{\text {chir }}$ values that, with their low precision, could not be used quantitatively.

The results can be explained by the unusually high metal affinity of Arg. When the trimer ion is fragmented, no (denoted as ND in Table 1) or low dimer ion signal for the ion containing the non-Arg component results in the inability to measure branching ratios or unacceptable precision, respectively. His, having basicity (and likely, metal ion affinity) similar to that of Arg, can effectively compete for $\mathrm{Ca}^{\mathrm{II}}$, whereas the other amino acids cannot. However, the fact that a trimer ion could be isolated for all of these analyte/reference systems indicates a less skewed degree of metal ion affinity during the condensed-phase (in solution or during the ESI process) production of this ion form. Also interesting to note is the lack of reciprocity in the measured $R_{\text {chir }}$ values for discrimination of Arg enantiomers by the different His and Glu reference conformers. This phenomenon has been reported previously for systems containing His, but is, without further investigation, currently inexplicable [21, 29].

Results provided from the incorporation of the Cterminal amidated Arg derivatives also provide information regarding the formation of the diastereomeric trimer ion form. When Arg- $\mathrm{NH}_{2}$ and Ac-Arg- $\mathrm{NH}_{2}$ are employed as references, no trimer ion (denoted as NT in Table 1) is formed. This was consistent for all metal ions screened. Such a result has two implications: (1) formation of the specific trimeric ion form of interest $\left(\mathrm{A}_{\mathrm{X}} /\right.$ Ref/M, 1:2:1), likely involves the coordination of a deprotonated carboxyl group from the reference to the metal ion; and (2) a carboxyl group is needed to provide one of the coordination ligatures for the bicoordinate reference molecule. Figure 1 shows general arrangements of the coordinating ligands for all diastereomeric cluster ion forms investigated in these experiments. Other more detailed graphics, which conjecture as to the arrangement of specific functional moieties in metal-mediated trimer ions have been published, but only for simple amino acids which lack functional units outside of the $\alpha$-amine and carboxylic acid group [5, 34]. The results of this experiment seem to indicate that an arrangement, with carboxyl groups possibly binding one or both of the single coordinate species, and a 
(a)

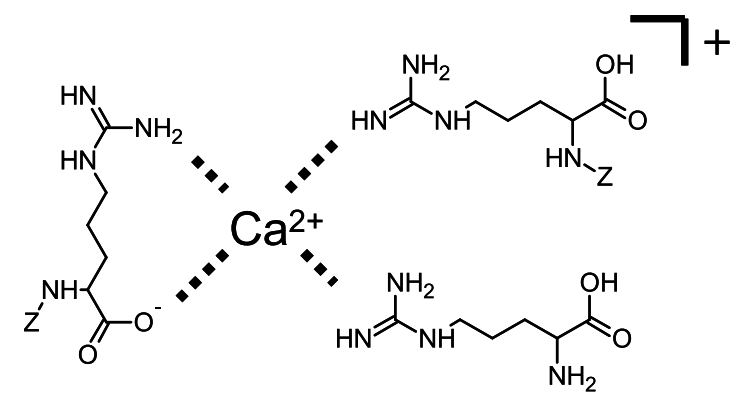

(b)

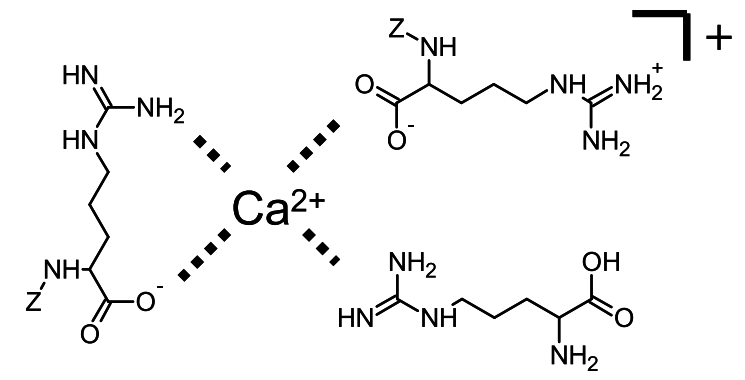

(c)

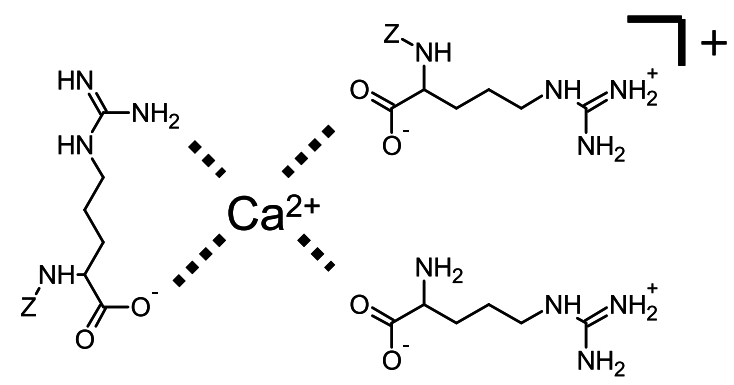

Figure 3. Idealized $\mathrm{Ca}^{2+}$-mediated trimer ion structures for enantiomeric discrimination of Arg by Z-Arg.

carboxyl and complimentary group binding the bicoordinate species, are possible. The complementary group (the second ligature in the bicoordinate ligand), however, in the case of Arg and Arg-derivatives is likely the guanidinium group, rather than the amino group commonly depicted, for simpler amino acid systems. This is confirmed by the inability of other amino acids to effectively compete against Arg in these experiments. Figure 3 shows an idealized schematic for Z-Arg employed as a reference for discriminating Arg as an analyte. Although it would be difficult to unequivocally determine which form is more prominent, Structure B appears to be less hindered by space-charge effects around the $\mathrm{Ca}^{\mathrm{II}}$ ion.

\section{Quantitative Analysis}

To demonstrate the applicability of the KM and the CR method for quantitative analysis of Arg enantiomers, calibration curves using sample mixtures of defined enantiomeric excess were constructed using Z-(R)-Arg as a reference and $\mathrm{Ca}^{\mathrm{II}}$ metal mediation. Quantitative discrimination in the KM can be achieved by relating the relative branching ratio to the difference in free energy change of the system by the kinetic method expression, given in eq 4 [39]:

$$
\ln \mathrm{R}=\Delta(\Delta \mathrm{G})_{\mathrm{KM}} / R T_{\text {eff. }} .
$$

Here, $R$ is the branching ratio as given previously, $R$ is the gas constant, $T_{\text {eff }}$ is the effective temperature of the activated trimeric cluster (fixed by a constant excitation amplitude during dissociation), and $\Delta(\Delta \mathrm{G})_{\mathrm{KM}}$ is the difference in free energy change for each pathway of dissociation for the diastereomeric trimeric clusters to the two dimeric clusters (shown previously in Figure 1) given by the KM. A nearly identical expression can be constructed for the CR method [19]:

$$
\ln (\mathrm{CR})=\Delta \mathrm{G}_{\mathrm{CR}} / R T_{\mathrm{eff}}
$$

where $\Delta G_{C R}$ measures the free energy change for the dissociation pathway to only the analyte-containing dimeric ion (i.e., the free energy of the reaction $\left[\mathrm{M}\left(\mathrm{A}_{\mathrm{X}}\right)\left(\operatorname{Ref}_{2}\right)-\mathrm{H}\right]^{+} \rightarrow\left[\mathrm{M}\left(\mathrm{A}_{\mathrm{X}}\right)(\mathrm{Ref})-\mathrm{H}\right]^{+}+$Ref). Each expression from eqs 4 and 5 can be related to each of the pure enantiomeric compounds. Using the $\mathrm{KM}$ as an example, if a binary mixture is used, where the enantiomeric excess is given by ee, a combined expression for $\Delta(\Delta \mathrm{G})_{\mathrm{KM}}$ can be given as follows:

$$
\begin{aligned}
\Delta(\Delta \mathrm{G})_{\mathrm{KM}} & =\text { ee }\left[\Delta(\Delta \mathrm{G})_{\mathrm{R}}\right]+(1-\text { ee })\left[\Delta(\Delta \mathrm{G})_{\mathrm{S}}\right] \\
& =\Delta(\Delta \mathrm{G})_{\mathrm{S}}+\left[\Delta(\Delta \mathrm{G})_{\mathrm{R}}-\Delta(\Delta \mathrm{G})_{\mathrm{S}}\right] \text { ee; }
\end{aligned}
$$

where $\Delta(\Delta G)_{R}$ and $\Delta(\Delta G)_{S}$ refer to the pure enantiomers, $A_{R}$ and $A_{S}$. By then combining eqs 4 and 6 , one obtains a convenient linear relationship relating the enantiomeric excess to the natural logarithm of the branching ratio, as shown in eq 7 :

$$
\begin{aligned}
\ln R & =\Delta(\Delta \mathrm{G})_{S} / R T_{\text {eff }}+\left[\Delta(\Delta \mathrm{G})_{\mathrm{R}}-\Delta(\Delta \mathrm{G})_{\mathrm{S}} / R T_{\text {eff }}\right] \text { ee } \\
& =\ln \mathrm{R}_{\mathrm{S}}+\left[\ln \mathrm{R}_{\text {chir }}\right] \text { ee. }
\end{aligned}
$$

This equation can be used to construct a calibration curve (ln $\mathrm{R}$ versus ee) with mixtures of known enantiomeric excess. For the relationship given in eq $7, \ln R_{\text {chir }}$ becomes the slope of the curve and $\ln R_{S}$, or the branching ratio determined for the pure enantiomer $A_{S}$ becomes the y-intercept. A similar derivation can be made for the CR method [19]. Similarly, a plot of $\ln$ (Int $\left.\left[\mathrm{M}\left(\mathrm{A}_{\mathrm{X}}\right)(\operatorname{Ref})-\mathrm{H}\right]^{+} / \operatorname{Int}\left[\mathrm{M}\left(\mathrm{A}_{\mathrm{X}}\right)\left(\operatorname{Ref}_{2}\right)-\mathrm{H}\right]^{+}\right)$, where Int denotes mass spectral intensity, versus ee yields a straight line which can be applied for quantitative enantiomeric analysis.

Figure 4 shows the quantitative results for the analysis of Arg enantiomers by Z-(R)-Arg, using $\mathrm{Ca}^{\mathrm{II}}$ as the metal mediator for the KM (Part a) and the CR method (Part b). Enantiomeric mixtures of 100/0, 98/2, 90/10, 50/50, 10/90, 2/98, and 0/100 [(R)-Arg/(S)-Arg], with $\mathrm{Ca}^{\mathrm{II}}$ in the same concentration as the screening experiments above, were prepared and run to construct the 
(a)

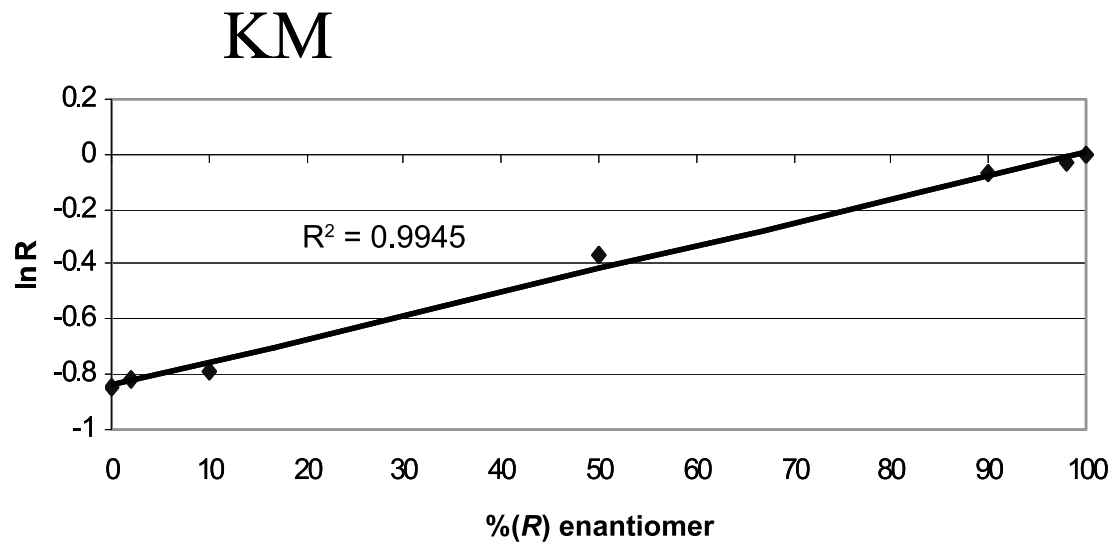

(b) $\mathrm{CR}$

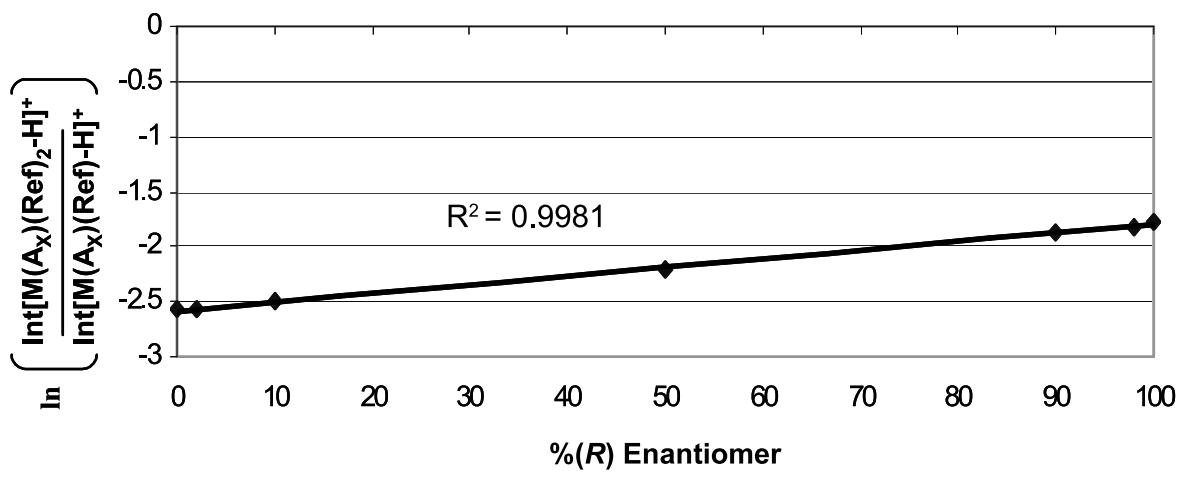

Figure 4. Quantitative determination of Arg enantiomers using Z-(R)-Arg as the chiral reference and $\mathrm{Ca}^{\mathrm{II}}$ metal mediation (for conditions, see Experimental section).

calibration curves. The results indicate good and comparable linearity for the two methods $\left(R^{2}=0.9945\right.$ for $\mathrm{KM}$ and $\mathrm{R}^{2}=0.9981$ for $\mathrm{CR}$ analysis). Each technique, in the context of this particular reference/analyte/metal system and the selected diastereomeric ions, provides acceptable results for quantitative analysis of Arg enantiomers.

\section{Conclusions}

This series of experiments was devised to develop and compare tandem-MS based approaches for chiral discrimination of Arg and Arg derivative enantiomers. Differentiation of Arg enantiomers in these experiments was achieved through analyte incorporation in the formation and dissociation of $\mathrm{Ca}^{\mathrm{II}}$-mediated diastereomeric trimer clusters, primarily using enantiomerically pure N-blocked Arg derivatives as chiral reference molecules. Results obtained using other commonly employed transition metal ions were inferior, showing no chiral resolution. The enantiomer discrimination values were higher than previously reported [34]. Additionally, the use of N-blocked Arg derivatives, having a similar metal ion affinity to unblocked Arg, offers an improved approach to discrimination of Arg enantiomers. Reciprocally, this method provides a means for chiral discrimination of enantiomers of N-blocked Arg derivatives with similar quantitative capability.

Other amino acids of varying functionality were used to qualitatively study their affinity for $\mathrm{Ca}^{\mathrm{II}}$ relative to Arg. Only His, a similarly basic amino acid, showed the ability to effectively compete for sites on the metal ion during stereoselective trimer ion dissociation. However, the fact that trimer ions were formed in the presence of Arg indicates a large difference in metal affinity for the amino acids between the condensed phase (when the trimer is formed in solution phase or during ESI droplet evaporation) and the gas phase (when the trimer is dissociated). Additionally, experiments incorporating enantiomerically pure C-blocked Arg references indicate the necessity of the carboxyl functional unit to form the described $\mathrm{KM}$ trimer ion complex.

The comparison of the $\mathrm{KM}$ approach to analysis using the CR method returned mixed results. Some systems, particularly those where a large disparity in metal ion affinity existed between analyte and reference (in this CR set-up, where the analyte metal ion affinity is greater than the reference metal ion affinity), showed improved results with the CR method over the KM. Quantitatively, for the chiral discrimination of Arg enantiomers using enantiomerically 
pure Z-Arg as chiral reference, the methods returned similar results. Overall, the KM is expected to perform with higher accuracy where applicable, due to the incorporation of a reference dissociation channel in the calculation.

In summary, the KM and the CR offer good quantitative methods for enantiomeric excess analysis. Application of KM has been cited to allow for ee determination at the 98:2 or 2:98 level, and these results, similarly for the CR method, seem to corroborate this assessment. The simplicity, precision, and robustness of the approaches make them ideal MS-based "chiral (and enantioselective) resolution methods". In general, their possible application in a diverse number of thermodynamic and steric determinations, and their mutual complementarity, makes them attractive for further extension to analytically interesting systems.

\section{Acknowledgments}

The authors acknowledge and greatly appreciate support for this research provided by the FWF (Austrian Science Fund, project no. P15482). The authors also thank Dr. Norbert Maier for help with the synthesis and purification strategies used in this work.

\section{References}

1. Pasteur, L. Mémoire sur la relation qui peut exister entre la forme cristalline et la composition chimique, et sur la cause de la polarisation rotatoire. Comp. Rend. Paris 1848, 26, 535-538.

2. Maier, N. M.; Franco, P.; Lindner, W. Separations of enantiomers: Needs, challenges, perspectives. J. Chromatogr. A 2001, 906, 3-33.

3. Süßmuth, R. D.; Jung, G. Impact of mass spectrometry on combinatorial chemistry. J. Chromatogr. B 1999, 725, 49-65.

4. Daniel, J. M.; Friess, S. D.; Rajagopalan, S.; Wendt, S.; Zenobi, R. Quantitative determination of noncovalent binding interactions using soft ionization mass spectrometry. Int. J. Mass Spectrom. 2002, 216, 1-27.

5. Filippi, A.; Giardini, A.; Piccirillo, S.; Speranza, M. Gas-phase enantioselectivity. Int. J. Mass Spectrom. 2000, 198, 137-163.

6. Sawada, M. Chiral recognition detected by fast atom bombardment mass spectrometry. Mass Spectrom. Rev. 1997, 16, 73-90.

7. Wu, L.; Tao, A.; Cooks, R. G. Kinetic method for the simultaneous chiral analysis of different amino acids in mixtures. $J$. Mass Spectrom. 2003, 38, 386-393.

8. Augusti, D. V.; Carazza, F.; Augusti, R.; Tao, W. A.; Cooks, R. G. Quantitative chiral analysis of sugars by electrospray ionization tandem mass spectrometry using modified amino acids as chiral reference compounds. Anal. Chem. 2002, 74, 3458-3462.

9. Sawada, M.; Takai, Y.; Yamada, H.; Hirayama, S.; Kaneda, T.; Tanaka, T.; Kamada, K.; Mizooku, T.; Takeuchi, S.; Ueno, K.; Hirose, K.; Tobe, Y.; Naemura, Y. Chiral recognition in hostguest complexation determined by the enantiomer-labeled guest method using fast atom bombardment mass spectrometry. J. Am. Chem. Soc. 1995, 117, 7726-7736.

10. Sawada, M.; Takai, Y.; Yamada, H.; Nishida, J.; Kaneda, T.; Arakawa, R.; Okamoto, M.; Hirose, K.; Tanaka, T.; Naemura, K. Chiral amino acid recognition detected by electrospray ionization (ESI) and fast atom bombardment (FAB) mass spectrometry (MS) coupled with the enantiomer-labeled guest method. J. Chem. Soc. Perkin Trans. 2 1998, 3, 701-710.
11. Ramirez, J.; He, F.; Lebrilla, C. B. Gas-phase chiral differentiation of amino-acid guests in cyclodextrin hosts. J. Am. Chem. Soc. 1998, 120, 7387-7388.

12. Dearden, D. V.; Dejsupa, C.; Liang, Y. J.; Bradshaw, J. S.; Izatt, R. M. Intrinsic contributions to chiral recognition: Discrimination between enantiomeric amines by dimethyldiketopyridino-18-crown-6 in the gas phase. J. Am. Chem. Soc. 1997, 119, 353-359.

13. Grigorean, G.; Lebrilla, C. B. Enantiomeric analysis of pharmaceutical compounds by ion/molecule reactions. Anal. Chem. 2001, 73, 1684-1691.

14. Speranza, M. Enantioselectivity in gas-phase ion-molecule reactions. Int. J. Mass Spectrom. 2004, 232, 277-317.

15. Guo, J.; Wu, J.; Siuzdak, G.; Finn, M. G.Measurement of enantiomeric excess by kinetic resolution and mass spectrometry. Angew. Chem. Int. Ed. 1999, 38, 1755-1758.

16. Dang, T. T.; Pedersen, S. F.; Leary, J. A. Chiral recognition in the gas phase: Mass spectrometric studies of diastereomeric cobalt complexes. J. Am. Soc. Mass Spectrom. 1994, 5, 452-459.

17. Smith, G.; Leary, J. A. Differentiation of diastereomeric nickel(II) N-glycoside complexes using tandem mass spectrometry and kinetic energy release measurements. J. Am. Chem. Soc. 1996, 118, 3293-3294.

18. Carlesso, V.; Fournier, F.; Tabet, J.-C. Stereochemical differentiation of four monosaccharides using transition metal complexes by electrospray ionization/ion-trap mass spectrometry. Eur. J. Mass Spectrom. 2000, 6, 421-428.

19. Yao, Z.-P.; Wan, T. S. M.; Kwang, K.-P.; Che, C. T. Chiral analysis by electrospray ionization mass spectrometry/mass spectrometry. 1. Chiral recognition of 19 common amino acids. Anal. Chem. 2000, 72, 5383-5393.

20. Yao, Z.-P.; Wan, T. S. M.; Kwang, K.-P.; Che, C. T. Chiral analysis by electrospray ionization mass spectrometry/mass spectrometry. 2. Determination of enantiomeric excess of amino acids. Anal. Chem. 2000, 72, 5394-5401.

21. Tao, W. A.; Zhang, D.; Nikolaev, E. N.; Cooks, R. G. Cooper (II)-assisted enantiomeric analysis of D,L-amino acids using the kinetic method: Chiral recognition and quantification in the gas phase. J. Am. Chem. Soc. 2000, 122, 10598-10609.

22. Tao, W. A.; Cooks, R. G. Chiral analysis by MS. Anal. Chem. 2003, 75, 25A-31A.

23. Cooks, R. G.; Kruger, T. L. Intrinsic basicity determination using metastable ions. J. Am. Chem. Soc. 1977, 99, 1279-1281.

24. Mirza, S. P.; Krishna, P.; Prabhakar, S.; Vairamani, M.; Giblin, D.; Gross, M. L. The kinetic method reveals secondary deuterium effects and the proton affinity and gas-phase basicity of glycine and alanine methyl esters. Int. J. Mass Spectrom. 2003, 230, 175-183.

25. Green-Church, K. B.; Limbach, P. A. Mononucleotide gasphase proton affinities as determined by the kinetic method. J. Am. Soc. Mass Spectrom. 2000, 11, 24-32.

26. Hahn, I.-S.; Wesdemiotis, C. Protonation thermochemistry of $\beta$ alanine. An evaluation of proton affinities and entropies determined by the extended kinetic method. Int. J. Mass Spectrom. 2003, 222, 465-479.

27. Nemirovsky, O. V.; Gross, M. L. Intrinsic Ca2+ Affinities of peptides: Application of the kinetic method to analogs of calcium-binding site III of rabbit skeletal triponin c. J. Am. Soc. Mass Spectrom. 2000, 11, 770-779.

28. Wu, L.; Lemr, K.; Aggerholm, T.; Cooks, R. G. Recognition and quantification of binary and ternary mixtures of isomeric peptides by the kinetic method: Metal ion and ligand effects on the dissociation of metal-bound complexes. J. Am. Soc. Mass Spectrom. 2003, 14, 152-160.

29. Schug, K. A.; Lemr, K.; Lindner, W. Isomeric discrimination of arginine-containing dipeptides using electrospray ionization- 
ion trap mass spectrometry and the kinetic method. J. Am. Soc. Mass Spectrom. 2004, 15, 840-847.

30. Kawano, T.; Nomura, M.; Nisikado, A.; Nakaya, Y.; Ito, S. Supplementation of L-arginine improves hypertension and lipid metabolism but not insulin resistance in diabetic rats. Life Sci. 2003, 73, 3017-3026.

31. Chromiak, J. A.; Antonio, J. Use of amino acids as growthhormone releasing agents by athletes. Nutrition 2002, 18, 657-661.

32. Shields, S. J.; Bluhm, B. K.; Russell, D. H. Fragmentation chemistry of $[\mathrm{M}+\mathrm{Cu}]+$ peptide ions containing an Nterminal arginine. J. Am. Soc. Mass Spectrom. 2000, 11, 626-638.

33. Tao, W. A.; Gozzo, F. B.; Cooks, R. G. Mass spectrometric quantitation of chiral drugs by the kinetic method. Anal. Chem. 2001, 73, 1692-1698.

34. Zhang, D.; Tao, W. A.; Cooks, R. G. Chiral resolution of Dand L-amino acids by tandem mass spectrometry of $\mathrm{Ni}(\mathrm{II})-$ bound trimeric complexes. Int. J. Mass Spectrom. 2001, 204, 159-169.
35. Hansch, C.; Leo, A.; Hoekman, D. Exploring QSAR: Hydrophobic, Electronic, and Steric Constants; American Chemical Society: Washington, D.C., 1995, p 217-324.

36. Nemirovsky, O. V.; Gross, M. L. Determination of calcium binding sites in gas-phase small peptides by tandem mass spectrometry. J. Am. Soc. Mass Spectrom. 1998, 9, 10201028.

37. Moorthy, A. K.; Singh, S. K.; Gopal, B.; Surolia, A.; Murthy, M. R. N. Variability of calcium binding to EF-hand motifs probed by electrospray ionization mass spectrometry. J. Am. Soc. Mass Spectrom. 2001, 12, 1296-1301.

38. Sinz, A.; Jin, A. J.; Zschörnig, O. Evaluation of the metal binding properties of a histidine-rich fusogenic peptide by electrospray ionization Fourier transform ion cyclotron resonance mass spectrometry. J. Mass Spectrom. 2003, 38, 1150-1159.

39. Cooks, R. G.; Wong, P. S. H. Kinetic method of making thermodynamic determinations: Advances and applications. Acc. Chem. Res. 1998, 31, 379-386. 